\title{
ГИПОКАЛИЕМИЧЕСКИЕ МИОПЛЕГИИ
}

\author{
Татьяна Геннадьевна Саковеи*, Энвер Ибрагимович Богданов
}

Казанский государственный медицинский университет

\begin{abstract}
Реферат
Остро развивающаяся генерализованная мышечная слабость может иметь различную этиологию. Среди всех ургетно госпитализированных больных гипокалиемию выявляют более чем у $20 \%$ пациентов. Обычно уменьшение концентрации калия в плазме крови не является фатальным, однако тяжёлая гипокалиемия может угрожать жизни больного, страдающего кардиоваскулярными заболеваниями и дыхательными нарушениями. Клинически гипокалиемия проявляется мышечными болями, слабостью, снижением перистальтики кишечника, запорами, выраженное снижение уровня калия в сыворотке крови может приводить к фатальному рабдомиолизу. Ключевые гормоны, регулирующие общий запас калия в организме и его нормальное перераспределение во внутри- и внеклеточной жидкости, - инсулин и катехоламины, взаимодействующие с $\beta$-адренергическими рецепторами. Регуляция уровня калия осуществляется инсулином по принципу обратной связи (гиперкалиемия стимулирует секрецию инсулина, снижение уровня калия её ингибирует). Уровень катехоламинов не определяется концентрацией калия в крови, однако влияет на его содержание. Гипокалиемия обычно бывает следствием истощения запасов калия в организме больного вследствие аномальной ренальной (приём тиазидных диуретиков, высоких доз глюкокортикоидов, антибиотиков, первичный гиперальдостеронизм вследствие аденомы, карциномы надпочечников, билатеральной гиперплазии надпочечников, синдром Лиддла, Бартера, Гительмана, дефицит $11 \beta-$ гидроксистерондегидрогеназы, 11ß-гидроксилазы, 17 $\alpha$-гидроксилазы, синдром Кушинга) и экстраренальной (интенсивная рвота, диарея вследствие избыточного приёма слабительных средств, сальмонеллёза, иерсиниоза, инфицирования вирусом иммунодефицита человека, опухолей кишечника, химиотерапии, лучевой терапии у больных онкологического профиля, целиакии, кишечного анастомоза, недостаток калия в пищевых продуктах) потери калия, трансмембранного перераспределения калия (семейный периодический гипокалиемический паралич, приём агонистов $\beta_{2}$-адренергических рецепторов, бронходилататоров, теофиллина). Гипокалиемические миоплегии приводят к фатальным осложнениям, что требует информированности врачей различного профиля о клинических проявлениях гипокалиемии, в основе которой лежат разные нозологии.
\end{abstract}

Ключевые слова: гипокалиемия, гипокалиемические параличи.

HYPOKALEMIC MYOPLEGIA T.G. Sakovets, E.I. Bogdanov. Kazan State Medical University, Kazan, Russia. Acute generalized muscle weakness may have a different underlying cause. Among all urgently admitted patients, hypokalemia is observed in more than $20 \%$ of cases. Usually blood potassium concentration decrease is not fatal, but severe hypokalemia can be life-threatening for the patient suffering from cardiovascular diseases and respiratory disorders. Hypokalemia manifests clinically by muscle pain, weakness, the intestinal motility disorders, constipation, marked decrease in serum potassium levels can lead to fatal rhabdomyolysis. The key hormones that regulate the total stock of potassium in the body and its normal redistribution of intra- and extracellular fluid are insulin and catecholamines, interacting with $\beta$-adrenergic receptors. Regulation of blood potassium level is provided by insulin and it is a feedback (hyperkalemia stimulates insulin secretion, decreased potassium levels inhibits it). The level of catecholamines is not determined by potassium concentration, however, it influences the serum potassium level. Hypokalemia is usually the result of potassium loss due to abnormal renal (treatment with thiazides, high doses of corticosteroids, antibiotics, primary hyperaldosteronism due to adenoma, adrenal carcinoma, bilateral adrenal hyperplasia, Liddle, Bartter's, Gitelman's syndromes, 11ß-hydroxysteroid dehydrogenase, 11 -hydroxylase, $17 \alpha$-hydroxylase deficiency, Cushing's syndrome) or extrarenal (intense vomiting, diarrhea due to excessive laxative use, salmonellosis, yersiniosis, HIV-infection, bowel tumors, chemotherapy, radiation therapy in cancer patients, celiac disease, jejunoileal bypass, a lack of potassium in food) potassium loss, an also a transmembrane shift of potassium (hypokalemic familial periodic paralysis, $\beta_{2}$-agonist, bronchodilators, theophylline intake). Hypokalemic myoplegia may lead to fatal complications, requiring doctors of various specialties' to know the clinical manifestations of hypokalemia, which may have different underlying cause. Keywords: hypokalemia, hypokalemic paralysis.

Острая гипокалиемическая миоплегия, характеризующаяся генерализованной острой мышечной слабостью, сопровождается низким содержанием калия в плазме крови (3,5 ммоль/л и менее). При небольшом снижении уровня калия в крови (до 3-3,5 ммоль/л) неврологическая симптоматика может не развиваться. Гипокалиемия обычно становится следствием истощения запасов калия в организме больного вследствие аномальной ренальной и экстраренальной потери калия, реже бывает результатом трансмембранного перераспределения калия [29]. Для уточнения причин гипокалиемии необходимо исследовать уровень суточного калия в моче [30].

Наиболее часто при гипокалиемии в орга-

Адрес для переписки: tsakovets@yandex.ru низме больного страдают нервно-мышечная, кардиоваскулярная и гастроинтенстинальная системы. При значительном дефиците калия, помимо слабости в нижних конечностях, распространяющейся по типу восходящего паралича, отмечают поражение краниальных (в том числе бульбарных) и респираторных скелетных мышц. На гипокалиемические параличи указывают внезапное начало миоплегического эпизода и арефлексия в отсутствие поражения сенсорной сферы, угнетения сознания и нарушения функций тазовых органов.

Гипокалиемия (менее 2,5 ммоль/л) может инициировать процесс рабдомиолиза. Скорость появления клинических признаков, как правило, коррелирует с тяжестью гипокалиемии. Смертность при гипокалиемической миоплегии 
определяется фатальными сердечными аритмиями, у больных, как правило, страдающих патологией кардиоваскулярной системы (ишемической болезнью сердца, хронической сердечной недостаточностью, гипертрофией левого желудочка), дыхательной недостаточностью [19]. Опасна гипокалиемия при приёме сердечных гликозидов, так как повышается связывание сердечных гликозидов с Na-K-АТФазой кардиомиоцитов (на которую они оказывают ингибирующее влияние, следовательно, содержание калия внутри клетки снижается, а натрия - возрастает). Это вместе с другими механизмами приводит к аритмии (от желудочковой экстрасистолии до мерцания желудочков) и требует активных лечебных мероприятий, направленных на восстановление содержания калия в сыворотке крови. К типичным изменениям электрокардиограммы (ЭКГ) при гипокалиемии относятся инверсия и уплощение зубца $T$, проявление $U$-волн, депрессия сегмента $S T$. Необходимо учитывать, что выраженность изменений на ЭКГ не всегда коррелирует со степенью гипокалиемии. Гипокалиемия различной этиологии обусловливает повышение артериального давления, так как в почках не ограничивается реабсорбция натрия.

Ключевые гормоны, регулирующие общий запас калия в организме и его нормальное перераспределение во внутри- и внеклеточной жидкости, - инсулин и катехоламины, взаимодействующие с $\beta$-адренергическими рецепторами. Как инсулин, так и катехоламины способствуют интрацеллюлярному обратному захвату калия путём активации Na-K-АТФазы. Высокое соотношение концентрации калия во вне- и внутриклеточной жидкости (38:1) поддерживается благодаря действию Na-K-AТФазы, активно переносящей ионы калия в клетку, а ионы натрия из неё (в соотношении 2:3). Вследствие активно го выведения натрия из клеток Na-K-AТФазой 85-90\% всего натрия, содержащегося в организме, находится во внеклеточной жидкости и по этой причине определяет её объём. Регуляция уровня калия осуществляется инсулином по принципу обратной связи (гиперкалиемия стимулирует секрецию инсулина, снижение уровня калия её ингибирует). Уровень катехоламинов не определяется концентрацией калия в крови применение $\beta$-адреноблокаторов увеличивает уровень калия в крови, использование агонистов $\beta$-адренорецепторов обусловливает возникновение гипокалиемии. Синтез Na-K-АТФазы стимулируется гормонами щитовидной железы, что может определять развитие гипокалиемии при избытке тиреоидных гормонов.

\section{Причины гипокалиемических миоплегий}

Семейный периодический гипокалиемический паралич (СПГП) - обратимое состояние, патогенез СПГП неизвестен [2]. Гипокалиемия при СПГП бывает следствием перераспределения калия с повышением его концентрации в мы934 шечных клетках, что является следствием наследственного дефекта клеточной мембраны с нарушением функционирования вольтаж-зависимых кальциевых каналов, чувствительных к дигидропиридину [15]. Наследуется СПГП по аутосомно-доминантному типу в двух третях случаев, в оставшейся трети случаев СПГП носит спорадический характер. Клинические проявления СПГП возникают в раннем детском возрасте, очень редко - после 25 лет, преимущественно у мужчин. Гипокалиемические атаки возникают с различной частотой (от 1 раза в неделю до 1 раза в год), их длительность составляет от 3-4 ч до нескольких дней. Диагноз СПГП устанавливают при наличии гипокалиемии, сопровождающейся генерализованной мышечной слабостью, которая может быть спровоцирована одновременным введением инсулина и декстрозы (глюкозы), пребыванием на холоде. Дифференциальную диагностику проводят с семейным гиперкалиемическим параличом, семейным нормокалиемическим параличом, врождённой парамиотонией, врождённой миотонией.

С лечебной целью применяют препараты калия в дозе 0,2-0,4 ммоль/кг внутрь с интервалом 15-30 мин под контролем ЭКГ и уровня калия в плазме крови. В случае нарушения функции глотания или рвоты калия хлорид вводят внутривенно [25]. Внутривенной инфузии декстрозы (глюкозы) необходимо избегать ввиду повышения внутриклеточного уровня калия с усугублением гипокалиемии. Необходим профилактический приём спиронолактона или ацетазоламида [20].

Тиреотоксический периодический паралич (ТПП) - наиболее частый вариант периодических параличей. До 90\% всех случаев ТПП зарегистрировано в Азии [34], однако он встречается также и в других популяциях [18, 25, 31]. Указанная нозология по частоте значительно преобладает у мужчин. Начало миоплегии либо совпадает с дебютом клинических проявлений тиреотоксикоза, либо отсрочено на 20-40 дней [7]. Возникновению гипокалиемического паралича может способствовать нарушение функционирования калиевых [42] и/или кальциевых [4] каналов.

Очень редко встречаются семейные формы ТПП. У больных с эутиреозом эпизодов генерализованной мышечной слабости не бывает. В случае рецидива тиреотоксикоза отмечают возврат ТПП [34].

Необходимо тщательное обследование больных, так как тиреотоксикоз в половине случаев протекает субклинически, что в сочетании с неврологическими и лабораторными признаками, сходными с таковыми при СПГП, создаёт трудности в дифференциальной диагностике ТПП $[27,40]$. Коррекция тиреотоксикоза в сочетании с исключением провоцирующих факторов (физического напряжения, приёма алкоголя) приводит к нивелированию миоплегических эпизодов. Применение препаратов калия обусловливает ре- 
гресс генерализованной мышечной слабости [32]. Тяжесть и частоту миоплегических атак снижает применение $\beta$-адреноблокаторов [11].

При отравлении барием развиваются гипокалиемические параличи. Первые случаи были отмечены в китайском регионе Па Пинг (болезнь Па Пинг). Гипокалиемия развивается в случае приёма внутрь столовой соли, загрязнённой солями бария, продуктов, содержащих карбонат бария (родентицид) [37], случайного попадания в пищу яда для крыс, при авариях на заводах, суицидальных попытках, ингаляции паров карбоната бария [8]. Ятрогенные эпизоды миоплегии возникают при ошибочном применении растворимых солей бария вместо нерастворимых в воде солей. Все растворимые соли бария токсичны. Механизм действия этих солей заключается в том, что ионы бария, имея одинаковый радиус с ионами калия, конкурируют с ним в биохимических процессах, нарушают деполяризацию мембраны [28]. Отравление препаратами бария вызывает рвоту и диарею, которые усиливают гипокалиемию. Генерализованная мышечная слабость регрессирует после нивелирования связи бария с калиевыми каналами и его почечной экскреции.

С лечебной целью проводят внутривенную инфузию препаратов калия. Применяют также внутривенное введение натрия сульфата, которое, однако, в ряде случаев может приводить к преципитации бария в почечных канальцах и быть причиной почечной недостаточности [44]. При пероральном отравлении растворимыми солями бария приём натрия сульфата внутрь превращает их в не абсорбирующиеся в желудочно-кишечном тракте соли. Эффективно также применение магния сульфата внутривенно или внутрь.

Гипокалиемические параличи могут возникать вследствие повышенной экскреции калия при нарушении функций почек.

Часто причиной генерализованной мышечной слабости становится ренальный тубулярный ацидоз, как 1-го (дистальный тубулярный ацидоз), так и 2-го (проксимальный тубулярный ацидоз) типа. Первый может быть исходом медуллярной кистозной болезни почек [27], синдрома Шёгрена [12], отравления толуолом [6].

Выраженность гипокалиемии прямо не коррелирует со степенью ацидоза, отражая преимущественно количество принимаемого с пищей калия и натрия, а также концентрацию в крови альдостерона. Фатальный уровень калия (менее 2 ммоль/л) в крови возможен при нелеченом тубулярном почечном ацидозе. Причиной гипокалиемического паралича также могут быть синдром Фанкони с гиперхлоремическим метаболическим ацидозом, водная интоксикация, нефротический синдром, диуретическая фаза острого ренального ацидоза, синдромы Лиддла, Бартера [3], Гительмана, уретеросигмостомия.

Генетические нарушения, определяющие дефект ионных транспортёров, встречаются редко. При синдроме Лиддла и дефиците 11ß-гидроксистерондегидрогеназы увеличивается реабсорбция ионов натрия в почках, что обусловливает возникновение синдрома псевдогиперальдостеронизма (артериальной гипертензии и гипокалиемии) при нормальном или сниженном уровне альдостерона. При двух других генетических расстройствах мутации приводят к частичному или полному нарушению реабсорбции хлоридов в восходящем колене петли Генле (синдром Бартера) [46], дистальном отделе канальца (синдром Гительмана) с проявлениями гипокалиемии без артериальной гипертензии.

Лечение - коррекция гипокалиемии. Ацидоз, возникающий при дистальном тубулярном ацидозе, необходимо корректировать [41]: назначение натрия гидрокарбоната уменьшает клинические проявления гипокалиемии. Целесообразно рекомендовать больным также длительное употребление в пищу продуктов, богатых калием. У пациентов с проксимальным тубулярным ацидозом попытки регулировать уровень бикарбонатов могут привести к нарастанию гипокалиемии [43].

Гипокалиемия почти всегда сопровождает метаболический алкалоз, причиной которого бывает истощение запасов хлоридов вследствие рвоты, гастрального шунта. При алкалозе возрастает секреция калия в дистальных канальцах и собирательных трубочках почек преимущественно в результате конкурентных взаимоотношений между ионами калия и водорода. При хлоридчувствительных формах алкалоза назначение хлоридов позволяет восполнить запас калия (при условии его достаточного приёма внутрь). В редких случаях алкалоз не зависит от уровня хлоридов и является следствием интраренальных нарушений, связанных с нарушением реабсорбции натрия в дистальных отделах нефрона. Наиболее частая причина тяжёлой гипокалиемии (менее 3,0 ммоль/л) - гиперальдостернизм.

Гипокалиемия при метаболическом ацидозе возможна при дистальном и проксимальном тубулярном ренальном ацидозе.

Гипокалиемические параличи при эндокринопаmиях. Синдром Кона, обусловленный альдостерон-синтезирующей аденомой надпочечников, и адренокортикальная карцинома [45] могут приводить к гипокалиемическому параличу. Удаление новообразования приводит к исчезновению мышечной слабости [33], в случае невозможности оперативного лечения рекомендуют приём спиронолактона.

Гипокалиемия может возникать при синдроме Кушинга, однако уровень калия никогда не снижается так низко, как при гиперальдостеронизме.

Гипокалиемические параличи, индуцированные приёмом лекарственных препаратов, способствующих трансиеллюлярному перераспределению калия. $\mathrm{K}$ гипокалиемическому параличу может приводить использование $\beta$-симпатомиметиков: бронходилататоров, ингибиторов контрактильной активности матки [1]. Стандартная доза саль- 
бутамола (альбутерола) в небулайзере уменьшает уровень калия на 0,2-0,4 ммоль/л, вторая доза в течение часа обусловливает уменьшение содержание калия в крови на 1 ммоль/л в течение последующих 4 ч $[9,10]$. Ритодрин и тербуталин (ингибиторы сокращения матки) могут приводить в снижению уровня калия в крови на 2 ммоль/л в течение нескольких часов после их внутривенной инфузии.

Теофиллин и кофеин, не являясь симпатомиметиками, стимулируют высвобождение катехоламинов, способствующих усилению Na-K-АТФазной активности. Интоксикацию теофиллином почти всегда сопровождает тяжёлая гипокалиемия. Даже при приёме нескольких чашек кофе уровень калия снижается на 0,4 ммоль/л. Блокаторы кальциевых каналов увеличивают обратный захват калия клеткой, однако в обычных терапевтических дозах не приводят к нарушению калиевого обмена. Приём токсических доз верапамила в некоторых случаях может быть причиной тяжёлой гипокалиемии.

Гипокалиемия при приёме препаратов, способствующих потере калия. Наиболее частая причина гипокалиемии - диуретическая терапия. Как тиазидные, так и петлевые диуретики блокируют хлорид-ассоциированную реабсорбцию натрия с ингибированием хлорид-натриевого транспортёра и, как результат, увеличивают доставку натрия в собирательные трубочки, где его реабсорбция определяет экскрецию калия. Степень гипокалиемии прямо связана с дозой тиазидных диуретиков и прямо пропорционально зависит от количества натрия, потребляемого больным [22]. Применение фуросемида в комбинации с буметанидом и метолазоном определяет возникновение гипокалиемии независимо от количества вводимого калия. Гипокалиемия при использовании диуретиков часто, но не всегда ассоциируется с умеренным или выраженным алкалозом. Диуретик ацетазоламид увеличивает экскрецию калия в собирательных канальцах, обусловливает метаболический ацидоз в сочетании с гипокалиемией.

Флудрокортизон - минералокортикоид для приёма внутрь, повышает обратное всасывание ионов натрия, хлора и воды в почках, одновременно увеличивая экскрецию ионов калия и водорода. Глюкокортикоиды (преднизолон, гидрокортизон) прямо не влияют на выделение калия через почки, однако обладают неспецифическим влиянием на скорость его фильтрации в почечных канальцах [13]. При длительном приёме глюкокортикоидов отмечают незначительное снижение концентрации калия (0,2-0,4 ммоль/л).

Пенициллин и его синтетические аналоги при внутривенном введении в больших дозах способствуют увеличению почечной экскреции калия. Аминогликозиды, противоопухолевый препарат цисплатин, противовирусный препарат фоскарнет приводят к увеличению потери калия через почки, снижая уровень магния в крови [16].

936
Большие потери калия со стулом при приёме слабительных средств [5] и повторных клизмах определяют возникновение гипокалиемии, что иногда бывает сложно выявить, несмотря на тщательный сбор анамнеза.

Гипокалиемия, возникающая вследствие недостаточного поступления калия с пищей. В редких случаях при приёме калия менее 1 г/сут с пищей (25 ммоль/сут) развивается гипокалиемия из-за сохраняющейся скорости экскреции калия почками и истощения его запасов в организме [21].

Гипокалиемия вследствие экстраренальной потери калия возникает при целиакии, тропической спру [17], остром гастроэнтерите [36], мальабсорбции при синдроме укороченной тонкой кишки. При диарее количество калия в стуле уменьшается, однако за счёт нарастания потери жидкости потери этого иона становятся значительными [23].

Гипокалиемия, обусловленная различными причинами. Снижение запасов магния в организме, возникающее при недостаточном потреблении магния или его аномальной потере, приводит к уменьшению содержания внутриклеточного калия, что определяется частичной инактивацией Na-K-АТФ-азы, увеличением почечной экскреции калия [38]. Причины последнего явления до сих пор остаются неясными. Частое сочетание снижения уровня магния с гипокалиемией наблюдается при применении диуретиков, амфотерицина В, а также гиперальдостеронизме и диарее. Независимо от причин гипокалиемии при наличии дефицита магния (особенно при гипомагниемии менее 0,5 ммоль/л) затруднена коррекция сниженного уровня калия в крови.

Тяжёлая, плохо корригируемая гипокалиемия встречается при миелогенном монобластном или лимфобластном лейкозе и связана с повышенной экскрецией калия почками. В случае ремиссии лейкоза гипокалиемия разрешается.

При неконтролируемом сахарном диабете увеличение выделения глюкозы с мочой приводит к повышению осмотического диуреза, концентрации натрия в дистальном отделе нефрона, нарастанию выведения калия. Однако грубой гипокалиемии при длительной глюкозурии не наблюдают, так как дефицит инсулина препятствует повышению уровня интрацеллюлярного калия. В случае быстрой коррекции недостаточности инсулина быстро возникают клинические признаки ранее латентной гипокалиемии, особенно при диабетическом кетоацидозе в результате стимуляции контртранспорта $\mathrm{Na}^{+} / \mathrm{H}^{+}$и опосредованной этим активации Na-K-АТФазы.

У больных с алкогольным делирием иногда отмечают внезапное падение уровня калия в крови до 1 ммоль/л [14], что коррелирует с повышением концентрации в крови эпинефрина вследствие значительной $\beta_{2}$-адренергической стимуляции.

Гипокалиемия возможна при лечении тяжёлой пернициозной анемии (гематокрит менее $20 \%$ ) цианокобаламином (витамином $\mathrm{B}_{12}$ ) ввиду 
быстрого захвата калия растущими клетками.

Снижение уровня калия в крови отмечают при переливании замороженной эритроцитарной массы, вероятно, вследствие захвата калия этими клетками [39].

Псевдогиперальдостеронизм при приёме как лакрицы (глицирризиновой кислоты) при однократном или постоянном употреблении в дозе 100-200 мг в день, так и карбексонолона (деривата глицирризиновой кислоты) может определять возникновение гипокалиемического паралича [35]. В этих случаях в лечебных целях показаны диета с исключением глицирризиновой кислоты и возмещение дефицита калия.

\section{Принципы возмещения дефищита калия в крови}

Гипокалиемия тяжёлой степени - угрожающее жизни состояние, требующее ургентного лечения. Рекомендовано введение калия внутривенно не более 20 ммоль в час при постоянном мониторировании сердечного ритма с последующим приёмом внутрь препаратов калия в течение нескольких недель. Калия бикарбонат применяют для коррекции гипокалиемии в сочетании с метаболическим ацидозом, калия фосфат используют при дефиците фосфатов. В остальных случаях назначают калия хлорид (внутрь, внутривенно) как уникальное средство коррекции гипокалиемии различной этиологии. Эффективно применение калийсберегающих диуретиков (амилорида, спиронолактона). Однако их применение даже в комбинации с петлевыми или тиазидными диуретиками может приводить к фатальной гиперкалиемии, риск которой нарастает при сахарном диабете или почечной недостаточности, что требует мониторирования уровня калия в крови и функций почек. Наиболее эффективно применение диеты, богатой калием, в комбинации с приёмом калия хлорида или калийсберегающих диуретиков.

Важность своевременной диагностики миоплегий определяется быстрым угрожающим жизни прогрессированием дыхательной и сердечно-сосудистой недостаточности.

\section{ЛИТЕРАТУРА}

1. Смирнов И.И., Лобойко К.Н., Никонов В.В. Медикаментозная гипокалиемия // Мед. неотл. состояний. 2009. - T. 6, №25. - C. 1-10.

2. Alkaabia J.M., Mushtaqc A., Al-Maskarib F.N. Hypokalemic periodic paralysis: a case series, review of the literature and update of management // Eur. J. Em. Med. - 2010. Vol. 17. - P. 45-47.

3. Amirlak I., Dawson K.P. Barter syndrome: an overview // Q. J. Med. - 2000. - Vol. 93. - P. 207-215.

4. Au K.S., Yeung R.T.T. Thyrotoxic periodic paralysis: periodic variation in the muscle calcium pump activity // Arch. Neurol. - 1972. - Vol. 26. - P. 543-546.

5. Basser L.S. Purgatives and periodic paralysis // Med. J. Aust. - 1979. - Vol. 1. - P. 47-48.

6. Bennett R.H., Forman H.R. Hypokalemic periodic paralysis in chronic toluene exposure // Arch. Neurol. 1980. - Vol. 37. - P. 673.
7. Bergeron L., Sternbach G.L. Thyrotoxic periodic paralysis // Ann. Emerg. Med. - 1988. - Vol. 17. - P. 843-845.

8. Berning J. Hypokalemia of barium poisoning // Lancet. - 1978. - Vol. 1. - P. 110.

9. Bremner P., Burgess C., Beasley R. et al. Nebulized fenoterol causes greater cardiovascular and hypokalaemic effects than equivalent bronchodilator doses of salbutamol in asthmatics // Respir. Med. - 1992. - Vol. 86. - P. 419-423.

10. Burgess C.D., Flatt A., Siebers R. et al. A comparison of the extent and duration of hypokalaemia following three nebulized $\beta_{2}$-adrenoceptor agonists // Eur. J. Clin. Pharmacol. - 1989. - Vol. 36. - P. 415-417.

11. Conway M.J., Siebal J.A., Eaton R.P. Thyrotoxicosis and periodic paralysis: improvement with beta blockade // Ann. Intern. Med. - 1974. - Vol. 81. - P. 332-336.

12. Dowd J.E., Lipsky P.E. Sjogren's syndrome presenting as hypokalemic periodic paralysis // Arthritis Rheum. 1993. - Vol. 36, N. 2. - P. 1735-1738.

13. Edwards C.R.W., Walker B.R., Benediktsson R. et al. Congenital and acquired syndromes of apparent mineralocorticoid excess // J. Steroid. Biochem. Mol. Biol. - 1993. Vol. 45. - P. 1-5.

14. Elisaf M., Liberopoulos E., Bairaktari E. et al. Hypokalaemia in alcoholic patients // Drug. Alcohol. Rev. 2002. - Vol. 21. - P. 73-76.

15. Fontaine B., Vale-Santos J., Jurkat-Rott K. et al. Mapping of the hypokalemic periodic paralysis (Hypo PP) locus to chromosome 1q31-32 in three European families // Nat. Genet. - 1994. - Vol. 6. - P. 267-272.

16. Gearhart M.O., Sorg T.B. Foscarnet-induced severe hypomagnesemia and other electrolyte disorders // Ann. Pharmacother. - 1993. - Vol. 27. - P. 285-289.

17. Ghosh D., Dhiman R.K., Kohli A. et al. Hypokalemic periodic paralysis in association with tropical sprue: a case report // Acta. Neurol. Scand. - 1994. - Vol. 90. - P. 371373.

18. Ghose R., Quail G., King R. et al. Hypokalemic paralysis in remote aboriginal communities // Aust. Fam. Physician. - 1996. - Vol. 25. - P. 1172-1173.

19. Ginnari F.J. Hypokaliemia // New Engl. J. Med. 1998. - Vol. 339, N. 7. - P. 451-458.

20. Griggs R.C., Engel W.K., Resnick J.S. Acetazolamide treatment of hypokalemic periodic paralysis // Ann. Intern. Med. - 1970. - Vol. 73. - P. 39-48.

21. Hernandez R.E., Schambelan M., Cogan M.G. et al. Dietary $\mathrm{NaCl}$ determines the severity of potassium depletion induced metabolic alkalosis // Kidney Int. - 1987. Vol. 31. - P. 1356-1367.

22. Hoes A.W., Grobbee D.E., Peet T.M. et al. Do non-potassium-sparing diuretics increase the risk of sudden cardiac death in hypertensive patients? Recent evidence // Drugs. 1994. - Vol. 47. - P. 711-733.

23. Kassirer J.P., Schwartz W.B. The response of normal man to selective depletion of hydrochloric acid: factors in the genesis of persistent gastric alkalosis // Am. J. Med. 1966. - Vol. 40. - P. 10-18.

24. Jayasinghe K.S.A., Mohideen R., Sheriff M.H.R. et al. Medullary sponge kidney presenting with hypokalemic paralysis // Postgrad. Med. J. - 1984. - Vol. 60. - P. 303-304.

25. Kilpatrick R.E., Seiler-Smith S., Levine S.N. Thyrotoxic hypokalemic periodic paralysis: report of four cases in black American males // Thyroid. - 1994. - Vol. 4. P. 441-514.

26. Kruse B.A., Carlson R.W. Rapid correction of hypokalemia using concentrated intravenous potassium chloride infusions // Arch. Intern. Med. - 1990. - Vol. 150. - P. 613-617.

27. Kung A.W. Thyrotoxic periodic paralysis: a diagnostic challenge // J. Clin. Endocrinol. Metab. - 2006. Vol. 91. - P. 2490-2495.

28. Layzer R.B. Periodic paralysis and sodium-potassi- 
um pump // Ann. Neurol. - 1982. - Vol. 11. - P. 547-552.

29. Lin S.H., Halperin M.L. Hypokalemia: a practical approach to diagnosis and its genetic basis // Curr. Med. Chem. - 2007. - Vol. 14. - P. 1551-1565.

30. Lin S.H., Lin Y.F., Chen D.T. et al. Laboratory tests to determine the cause of hypokalemia and paralysis // Arch. Intern. Med. - 2004. - Vol. 164. - P. 1561-1566.

31. Liu Z., Braverman L.E., Malabanan A. Thyrotoxic periodic paralysis in a Hispanic man after the administration of prednisone // Endocr. Pract. - 2006. - Vol. 12. P. 427-431

32. Lu K.C., Hsu Y.J., Chiu J.S. et al. Effects of potassium supplementation on the recovery of thyrotoxic periodic paralysis // Am. J. Emerg. Med. - 2004. - Vol. 22. - P. 544-547.

33. Ma J.T.C., Wang C., Lam K.S.L. et al. Fifty cases of primary hyperaldosteronism in Hong Kong Chinese with a high frequency of periodic paralysis. Evaluation of techniques of tumor localization // Q. J. Med. - 1986. Vol. 61. - P. 1021-1037.

34. McFadzean A.J.S., Yeung R. Periodic paralysis complicating thyrotoxicosis in Chinese // BMJ. - 1967. Vol. 1. - P. 451-455.

35. Nielsen I. Life-threatening hypokalemia caused by liquorice ingestion // Lancet. - 1984. - Vol. 1. - P. 1305.

36. Orman R.A., Lewia J.B.J. Flaccid quadriparesis associated with Yersinia enterocolitis-induced hypokalemia // Arch. Intern. Med. - 1989. - Vol. 149. - P. 1193-1194.

37. Polson C.J., Green M.A., Lee M.R. Clinical toxicology, 3rd edn. - London: Pitman, 1984. - 495 p.
38. Pun K., Wong C., Tsui E.Y. et al. Hypokalemic periodic paralysis due to Sjogren's syndrome in Chinese patients // Ann. Intern. Med. - 1989. - Vol. 110. - P. 405-406.

39. Rao T.L.K., Mathru M., Salem M.R. et al. Serum potassium levels following transfusion of frozen erythrocytes // Anesthesiology. - 1980. - Vol. 52. - P. 170-172.

40. Rhee E.P., Scott J.A., Dighe A.S. Case 42012: a 37-year-old man with muscle pain, weakness, and weight loss // N. Engl. J. Med. - 2012. - Vol. 366. - P. 553-560.

41. Rowbottom S.J., Ray D.C., Brown D.T. Hypokalemic paralysis associated with renal tubular acidosis // Crit. Care Med. - 1987. - Vol. 15. - P. 1067-1068.

42. Ryan D.P., da Silva M.R., Soong T.W. et al. Mutations in potassium channel Kir2.6 cause susceptibility to thyrotoxic hypokalemic periodic paralysis // Cell. - 2010. Vol. 140. - P. 88-98.

43. Sebastian A., McSherry E., Morris R.C.J. Renal potassium wasting in renal tubular acidosis (RTA). Its occurrence in types 1 and 2 RTA despite sustained correction of systemic acidosis // J. Clin. Invest. - 1971. - Vol. 50. P. 667-668.

44. Wertherill S.F., Guarino M.J., Cox R.W. Acute renal failure associated with barium chloride poisoning // Ann. Intern. Med. - 1981. - Vol. 95. - P. 187-188.

45. Young W.F.Jr. The incidentally discovered adrenal mass // N. Engl. J. Med. - 2007. - Vol. 356. - P. 601-610.

46. Zelikovic I. Hypokalaemic salt-losing tubulopathies: an evolving story // Nephrol. Dial. Transplant. - 2003. Vol. 18. - P. 1696-700

\title{
РЕНТГЕНЭНДОВАСКУЛЯРНЫЕ МЕТОДЫ В ПРОФИЛАКТИКЕ И ЛЕЧЕНИИ АРРОЗИВНЫХ КРОВОТЕЧЕНИЙ У БОЛЬНЫХ С ДЕСТРУКТИВНЫМИ ФОРМАМИ ПАНКРЕАТИТА
}

\author{
Азат Минвагизович Зайнутдинов *, Игорь Сергеевич Малков, Алексей Альбертович Валеев
}

Казанская государственная медицинская академия

\section{Реферат}

В обзоре представлены методы рентгенэндоваскулярной хирургии в профилактике и лечении аррозивных кровотечений при деструктивном панкреатите. Освещена актуальность консервативной тактики лечения у больных с асептическими формами деструктивного панкреатита. Отмечено, что проблема выбора оптимального хирургического лечения у пациентов с панкреонекрозом в настоящее время не решена. Показана эффективность использования малоинвазивных способов лечения деструктивного панкреатита. Один из вариантов такого направления - рентгенэндоваскулярные методы лечения. Они включают внутриартериальную инфузию лекарственных средств и эмболизацию сосудов, кровоснабжающих поджелудочную железу, для профилактики и лечения гнойно-воспалительных процессов и аррозивных кровотечений из псевдоаневризм висцеральных артерий. Клиническим обоснованием селективного введения препаратов в чревную артерию при лечении деструктивного панкреатита служат исследования отечественных и зарубежных авторов. В обзоре указаны клинические случаи эмболизации висцеральных артерий (селезёночной, гастродуоденальной, левой толстокишечной, внутрипечёночной) как способ профилактики и экстренного лечения геморрагических осложнений при деструктивном панкреатите. Проблему аррозивных кровотечений при деструктивных панкреатитах на современном этапе можно адекватно решать в условиях специализированных хирургических стационаров, оснащённых операционной рентген-хирургической установкой и мультиспиральным компьютерным томографом.

Ключевые слова: кровотечения, острый панкреатит, псевдоаневризма, рентгенэндоваскулярные методы.

RADIO-ENDOVASCULAR METHODS IN PREVENTION AND TREATMENT OF ARROSIVE BLEEDINGS IN PATIENTS WITH DESTRUCTIVE PANCREATITIS A.M. Zainutdinov, I.S. Malkov, A.A. Valeev. Kazan State Medical Academy, Kazan, Russia. The review decribes the radio-endovascular methods in prevention and treatment of arrosive bleedings in destructive pancreatitis. The importance of non-surgical treatment in patients with aseptic forms of destructive pancreatitis is presented. It is stressed that the problem of an optimal surgical treatment choice in patients with pancreatolysis is not solved. Efficiency of minimally invasive surgery for treating destructive pancreatitis is shown. Radio-endovascular treatment methods, including intraarterial drug infusions and pancreatic arteries embolization to prevent and treat pancreatic inflammation and arrosive bleedings from visceral arteries aneurisms, are among such options. Researches of domestic and foreign authors give the clinical basis for selective drug introduction into coeliac artery in destructive pancreatitis treatment. 